\title{
OBLIGACIÓN POLÍTICA: ALGUNOS PROBLEMAS Y UN INTENTO DE SOLUCIÓN
}

Rex Martin

Universidad de Kansas

\section{Introducción}

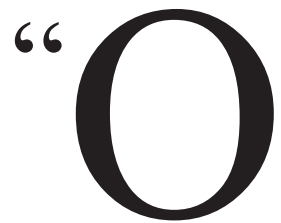

bligación política" es una noción muy amplia que abarca muchas cosas. Hay quien dice, por ejemplo, que el ciudadano tiene una obligación o un deber de votar. Otros han pretendido que los ciudadanos pueden tener un deber de servir a su país e incluso de luchar en su defensa. Sin embargo, la mayoría de la gente cuando habla de obligación política tiene en mente una cosa en particular: el deber de los ciudadanos de obedecer las disposiciones jurídicas [laws] en su propio país.

La cuestión que quiero discutir en este artículo es la de si la gente tiene en realidad buenas y justificables razones, que estén más allá del simple miedo al castigo, para comportarse de acuerdo con las disposiciones jurídicas. Y si es así, si están vinculados u obligados por esas razones para cumplir.

\section{Algunos argumentos estándar en favor de un deber de obedecer al Derecho}

Sócrates tenía que decidir si desobedecía una decisión injusta, pero legal; lo notable es que, en circunstancias que iban a costarle la propia vida, decidiera obedecer en base a lo que él consideraba sólidas razones. Sócrates creía que la gente tenía un deber moral de obedecer al Derecho. Un deber muy estricto fundado en un acuerdo que la gente ha hecho ${ }^{1}$.

Lo característico del argumento del acuerdo de Sócrates (en Critón) es que pone la cuestión en términos de justicia o moralidad. En nuestra propia tradición política hay un argumento parecido al socrático; pone el acento

${ }^{1}$ Para esta parte del Critón que contiene los argumentos socráticos contra la desobediencia al Derecho, véase Platón, Five Dialogues, trad. G.M.A. Grube (Indianapolis, IN, Hackett, 1981), págs. 52-56. 
no tanto en la moralidad de mantener los acuerdos, cuanto en la conexión entre, por un lado, un estado [government] legítimamente constituido y, por otro, un deber de los ciudadanos de obedecer las disposiciones jurídicas establecidas por ese estado. Se trata de una obligación estricta; se refiere a todas las disposiciones jurídicas y sólo puede ser ignorada, si es que puede serlo, en casos excepcionales.

Para esta teoría, normalmente asociada a Hobbes y a Locke en particular, un contrato (a veces llamado "consentimiento en el gobierno") sirve tanto para autorizar al estado a dictar disposiciones jurídicas como para sujetar a los individuos a una estricta obediencia. Sin embargo, en realidad, las teorías de Hobbes y Locke no son tan sencillas.

Locke sostiene que, en un cierto momento (esto es, cuando se alcanza la edad adulta y, de forma más o menos voluntaria, se permanece en el lugar en vez de ejercer el derecho a la emigración), la gente se convierte en miembro o partes de un particular cuerpo político. La principal función de tal cuerpo es crear una constitución o forma de gobierno y cabe presumir que se produce un consenso (en términos de Locke, una mayoría) entre los ciudadanos a propósito de en qué instituciones deben residir los principales poderes del gobierno (legislativo, ejecutivo, etc). Naturalmente, en opinión de Locke, si no hubiera consenso el cuerpo político se rompería, simplemente se desintegraría, y sólo podría mantenerse unido mediante un uso manifiesto y claramente impropio de la fuerza. A partir de ahí, desde estos dos supuestos (que se es miembro de un cuerpo político y que hay un gobierno constitucional fundado en el consenso) Locke concluye, como una cuestión de lógica, que cada ciudadano (cada miembro de una sociedad política así organizada) está estrictamente obligado a obedecer las disposiciones jurídicas debidamente establecidas por ese gobierno constitucional. De los dos supuestos anteriores más otro -que si las disposiciones jurídicas no fueran obedecidas ciertamente la gente retornaría a un indeseado estado de naturaleza- se sigue que cada miembro tiene la obligación estricta en cuestión. En breve, no se consiente (contrata, promete) en obedecer las disposiciones jurídicas; más bien, se consiente en ser miembro de un cuerpo político $\mathrm{y}$, a partir de este hecho, junto con otro u otros dos más, se sigue lógicamente que los ciudadanos tienen un deber estricto de obedecer las disposiciones jurídicas debidamente establecidas. Se está obligado como si de hecho se hubiera consentido expresamente en obedecer ${ }^{2}$.

\footnotetext{
${ }^{2}$ Para los principales puntos (cuerpo político, consenso constitucional, evitación del estado de naturaleza), véase Locke, Second Treatise, sec. 97; y también secs. 89, 95-96, 98-99. La justificación de Locke de la división de poderes del Estado se encuentra en Second Treatise, caps. 11 y 12. La discusión de Locke del consentimiento expreso, esto es, el consentimiento perma-
} 
Los teóricos del "contrato", igual que Sócrates con la idea de un acuerdo, construyen la relación de los ciudadanos con el estado y sus disposiciones jurídicas a partir de una analogía con algunos compromisos no políticos, tales como prometer, acordar, consentir, o firmar un contrato, cuyo sentido es crear obligaciones. Para todas estas teorías, el hecho del acuerdo o el acto de consentimiento es el que fundamenta la obligación de obedecer las disposiciones jurídicas.

Esta aproximación general presenta, en mi opinión, dos problemas principales. El primer problema es el de qué cuenta como consentimiento. Y el segundo problema es el de si el consentimiento así concebido puede realmente obligar a la gente a obedecer todas o casi todas las disposiciones jurídicas válidas simplemente porque han sido dictadas de manera correcta por un estado legítimo o efectivo.

¿Qué cuenta como consentimiento? Todos los teóricos consideran la mera residencia, la residencia permanente, durante la edad adulta. Hobbes añade el interesante retorcimiento de que contaría plenamente como consentimiento el caso de un residente que, bajo la amenaza de pena de muerte si no presta "consentimiento", inclinara su cabeza y entrara a vivir sometido a un conquistador. Mucha gente tiene reticencias para aceptar que la mera residencia continuada deba tener exactamente la fuerza de una promesa explícita y solemne, especialmente bajo la condición añadida por Hobbes.

Se ha sostenido que votar en una elección libre debería contar así. Podríamos considerar que votando usted se estaría comprometiendo a aceptar el resultado de la elección. Pero ¿por qué se comprometería usted a aceptar, estando obligado a aceptar, todas las disposiciones jurídicas que dictaran los elegidos? Algunas de esas disposiciones podrían ser absurdas, inconstitucionales o incluso perversas. Suponga que vota al bando perdedor. Su candidato no gana. Usted votó de este modo porque quería que una determinada disposición que considera mala no fuera aprobada. Y ahora el candidato contra el que usted votó, junto con otros, apoyan esa misma disposición. O suponga que se tratara de una disposición realmente perversa, como la disposicón de U.S. de 1850, que exigía que los esclavos evadidos fueran recapturados y devueltos a sus propietarios, y que usted era un votante del Estado de Massachussets al que no le gustaba nada esa disposición y que había votado a un candidato que se oponía, candidato que resultó elegido, votó en contra de esa disposición pero que fue derrotado en la sesión de la legislatura nacional. Estos ejemplos sugieren que la cuestión va algo más

nente o mantenido de los ciudadanos miembros, tiene lugar Second Treatise secs. 116-118; y su justificación y contraste con el consentimiento tácito, esto es, el consentimiento temporal de los visitantes, etc., se encuentra en Second Treatise, secs. 119-122. 
allá de decir simplemente que por votar en una elección general usted se está comprometido a aceptar esta disposición y se está obligando a obedecerla.

Pero ¿qué ocurriría con una promesa explícita y solemne, con un acuerdo fuerte de aceptar todas las disposiciones válidas y de estar obligado por ellas? ¿Funcionaría? No demasiado bien. Muchos ciudadanos nunca consentirían o acordarían obedecer las disposiciones del país en el que residen de forma que realmente pudiera contar. Por ejemplo, no todo el mundo (ciertamente no cada ciudadano) se ha comprometido en un acto de consentimiento significativo en Gran Bretaña, América o Canadá; de hecho relativamente poca gente lo ha hecho. En consecuencia, si se requiere un consentimiento real y fuerte, entonces la teoría del contrato no puede valer para la obligación de obedecer al Derecho en esos países.

Siempre podría replicarse: bien, si cada uno hubiera libre y explícitamente prometido (en un juramento solemne de algún tipo) obedecer las disposiciones de su propio país, esto podría seguramente contar. Pero incluso si una promesa de este tipo contase como consentimiento, podríamos todavía preguntar si tal consentimiento explícito vincularía o podría vincular a aquellos que hubieran formulado el juramento, si podría obligarles, a obedecer todas las disposiciones válidas del lugar simplemente porque se trata de disposiciones de ese país. La estricta obligación de obedecer las disposiciones ¿está fundada meramente en la existencia desnuda de un consentimiento de hacerlo así, o está fundada en cualesquiera buenas razones (excluyendo naturalmente el miedo al castigo) que uno pudiera tener para consentir en primer lugar?

Obviamente, si simplemente citamos las razones (pero sin un acto explícito de consentimiento de la gente implicada) entonces no contaremos con un consentimiento real como base de la obligación, contrariamente a lo que la teoría del contrato requiere ${ }^{3}$. A pesar de ello, supongamos que citamos ambos, el hecho de un explícito y amplio acuerdo en un país dado y las buenas razones para realizar el acuerdo. Se podría entonces cuestionar si el hecho del consentimiento explícito realmente añade algo a esas razones.

\footnotetext{
${ }^{3}$ La idea que acabo de sugerir -que puede haber buenas razones para que la gente actúe como si hubieran explícitamente consentido- es llamada a veces del consentimiento hipotético. Para una crítica importante de toda la aproximación del "consenso hipotético", véase Ronald Dworkin, "Justice and rigths" (originalmente publicado en 1973), reimpreso en Ronald Dworkin: Taking Rigths Seriously (Cambridge, MA:Harvard University Press, 1978); esp. págs. 150-158. Continúa habiendo sin embargo sofisticadas defensas del consentimiento hipotético (como representando la mejor mirada dentro de la teoría tradicional del consentimiento). Entre aquellos que yo particularmente recomiendo están Thomas Lewis, "On using the concept of hypothetical consent”, Canadian Journal of Political Science 22 (1989), y Cynthia Stark, "Hypothetical consent and justification", Journal of Philosophy 97 (2000).
} 
Si consideramos nuestra obligación de obedecer al Derecho de nuestro país como una obligación moral (a la manera de Sócrates en el Critón), entonces probablemente creamos también que muchas o la mayoría de esas disposiciones tienen un buen contenido moral (tales como la prohibición del asesinato, el secuestro, la violación o el asalto físico). Pero ¿no serían las prohibiciones contenidas en tales disposiciones las que (dado su buen contenido moral) controlasen nuestra conducta, incluso en el caso de que ellas no hubieran sido consignadas en el Derecho? En el mismo sentido, moralmente hablando, ellas controlarían nuestra conducta incluso si nunca hubiéramos explícita y solemnemente prometido obedecer las disposiciones del lugar.

La cuestión se hace más complicada cuando tomamos en consideración disposiciones que son moralmente indiferentes. Me inclinaría a pensar que una obligación moral sólo podría surgir en aquellos casos en los que un requerimiento del Derecho (p.e. el pago de un impuesto sobre la renta) pudiera mostrarse como necesario o sustancialmente importante para la continuidad de la capacidad del estado para estimular el cumplimiento por parte de la gente de las disposiciones que sí tienen un contenido moralmente bueno.

Cuando se enfrenta la cuestión de las disposiciones perversas (normas con un mal contenido moral, como la Ley del Esclavo Fugitivo antes mencionada, o las normas Nazis contra los judíos) me parece que la cuestión cambia de manera considerable en relación con los dos primeros casos (el de las disposiciones con un buen contenido moral y el de las disposiciones con contenido moral neutro). Creo que no es posible fundamentar el precepto moral de obediencia al Derecho sobre una base de indiferencia en relación a si las disposiciones son de contenido moral bueno o al menos aceptable en la mayoría de casos y a la larga. La presunción moral aquí actúa contra las disposiciones perversas, y esta presunción actuará contra cualquier obligación moralmente basada de obedecer tales disposiciones. Conforme al análisis que se ha hecho, una promesa de obedecer disposiciones perversas no podría ser justificada moralmente y una promesa de este tipo, incluso la de obedecer todas las disposiciones (justas o injustas), no tendría fuerza obligatoria.

La insatisfacción con la teoría del consentimiento ha llevado a teóricos políticos a tomar en consideración otros posibles fundamentos para la obligación de obedecer al Derecho. Así, basándose en un apropiado sentido de la gratitud, con frecuencia se alega que la recepción de beneficios le obliga a uno a mostrar la conducta apropiada correspondiente. Algunos han dicho (por ejemplo, Platón en el Critón) que cuando el beneficio proviene del estado, la conducta apropiada correspondiente consiste en obedecer las disposiciones jurídicas. 
Otros se han centrado en una versión especial de la teoría del beneficio, llamada del fair play, para establecer el deber de obedecer al Derecho. He aquí el cuadro que ellos presentan. La gente está comprometida en una actividad, una práctica o una empresa compartida que es ampliamente beneficiosa (como ahorrar agua en tiempo de sequía o reducir el consumo eléctrico para encarar un apagón). Los beneficios de esta actividad sólo pueden obtenerse si la mayoría de la gente participa, pero hacerlo acarrea ciertos costes para cada uno de ellos. La teoría del fair play alega que dada nuestra participación en esta práctica (en particular, a través de la recepción voluntaria de beneficios y de los costes que para otros tiene la provisión de estos beneficios) debemos devolver de alguna forma los beneficios recibidos. Ello a menudo se traduce en cumplir con las disposiciones jurídicas.

En el presente estudio no podremos someter a discusión los argumentos recién introducidos en favor de un deber generalizado, un deber de cada uno, de obedecer todas las disposiciones jurídicas. Será suficiente con decir que un buen número de teóricos políticos se han centrado en estos tres argumentos estándar -los basados en el consentimiento, en la gratitud por los beneficios y en el fair play - y han mostrado que cada uno de ellos fracasaba.

A partir de ahí, algunos han concluido que simplemente no hay obligación, no hay obligación moral, para cada uno de obedecer todas las disposiciones de su propio país. En efecto, para algunos al menos puede no haber una situación de obligación de obedecer cualquiera de las disposiciones ${ }^{4}$. Otros, a partir de la misma muestra, han concluido que ninguno de los argumentos estándar puede funcionar, pero que quedan otros abiertos, que una aproximación radicalmente diferente puede funcionar. $\mathrm{Y}$ algunos incluso han sugerido las principales líneas de esta aproximación ${ }^{5}$. Me inclino hacia esta segunda visión, convenientemente matizada.

${ }^{4}$ John Simmons, en su libro Moral Principles and Political Obligations (Princeton, NJ, Princeton, NJ, 1979) discute un número de tales bases: fidelidad o consentimiento (caps. 3 y 4), fair play (cap. 5), el deber natural de justicia de Rawls (cap. 6) y gratitud o reembolso (cap. 7); véase también págs. 15-16 y 54-55. Simmons llega a la conclusión que he identificado en el cap. 8 de su libro; muchos teóricos anarquistas se harían, naturalmente, eco de esta conclusión.

${ }^{5}$ John Horton, en su libro Political Obligation (Atlantic Highlands, NJ, Humanities Press International, 1992) examina estos mismos fundamentos en caps. 2 (sobre el consentimientro) y 4 (fair play y gratitud) y los encuentra deficientes. Sugiere que hay que tomar una aproximación básica diferente que es desarrollada en el capítulo final de su libro (cap. 6). Para una línea de ataque similar con una similar conclusión, véase Ronald Dworkin, Law's Empire (Cambridge, MA, Harvard University Press, 1986), cap. 6. 


\section{Algunos materiales para un nuevo comienzo}

Atendiendo a cómo empezar de nuevo, reparemos en primer lugar en un interesante punto de semejanza en las tres teorías estándar: todas tratan la obligación de obedecer al Derecho como una obligación principalmente moral y así los fundamentos que enfatizan son fundamentos característicamente morales. Las teorías estándar están interesadas en los fundamentos generales para obedecer al Derecho -fundamentos operativos en todas o casi todas las sociedades, fundamentos que podrían cubrir todos los Derechos o, posiblemente, todas las personas- y desdeñan las razones que son locales o características sólo de una particular sociedad (o tipo específico de sistema). Pero sus análisis, por su propia naturaleza, crean un profundo problema; no pueden mostrar que los deberes así generados -por referencia a estos fundamentos generales característicamente morales (fundamentos como el acuerdo o el consentimiento expreso o la gratitud por los beneficios recibidos o el fair play)- pueden ser deberes de toda la gente de un país determinado de obedecer todas las disposiciones de ahí. $\mathrm{O}$ así ha sido argumentado. La búsqueda de esta generalidad se ha mostrado inútil y nada fructífera.

También es necesario sacar a relucir una segunda característica de la aproximación estándar. Los fundamentos favoritos citados por esta aproximación tienen todos en común que invocan algún acto voluntario por parte del sujeto. Típicamente, los sujetos voluntariamente han prestado consentimiento o, en una alternativa, voluntariamente han recibido beneficios $\mathrm{o}$, en otra alternativa, conscientemente han participado en una práctica $\mathrm{o}$ actividad compartida de la que han recibido voluntariamente beneficios de muchos clases y en la que ahora se les llama a contribuir. Todos estos casos descansan sobre el mismo punto principal: tener una obligación implica que voluntariamente se ha tomado dicha obligación mediante algún tipo de transacción (moralmente aceptable).

Este pronunciado énfasis en la voluntariedad tal vez esté fuera de lugar. Se pueden tener deberes que no estén en absoluto basados en actos voluntarios. Por ejemplo, los menores (digamos, los adolescentes) pueden tener deberes hacia los padres que no estén fundados en transacciones voluntarias por parte de los jóvenes; muchos de los beneficios que han recibido no pueden ser vistos como voluntarios o como tomados voluntariamente (por ejemplo, el enorme número de beneficios que recibieron cuando eran infantes o muy pequeños). Aún más, es posible que la relación en la que están con sus padres sea importante debido a (o al menos en parte) los deberes que tienen.

Considérese ahora un caso paralelo. Los requerimiento de conducta que el Derecho impone sobre la gente se deben a veces al estatus que los individuos tienen en una determinada sociedad política (tales como posa- 
dero, patrono o, más típicamente, ciudadano). Otras veces, las directivas normativas de conducta que impone el Derecho están vinculadas al territorio y se imponen simplemente debido a acciones creadoras de normas de los funcionarios del estado. Estos requerimientos se diferencian de las obligaciones voluntarias en un número importante de aspectos. No implican necesariamente compromisos o determinadas transacciones cuya función es poner a un ciudadano específico bajo un determinado requerimiento; en los casos típicos no obligan a individuos definidos o nombrados (sino, más bien, a todos los ciudadanos). Por decirlo en pocas palabras, necesitamos ser capaces de argumentar que conforme a tales disposiciones se puede tener un deber, sin asumir que ese deber está ahí sobre la base de algún compromiso voluntario o transacción determinada, cuya función no es otra que la de poner al ciudadano específicamente bajo este requerimiento.

Hay otra dimensión de la importante cuestión de la voluntariedad que también debe ser mencionada. La mayoría de la gente en realidad son ciudadanos o miembros de sólo un país durante toda su vida. Nacen en un país y pasan su vida en él. Muchos otros, por sus propias razones, se unen a ellos y ciertamente echan suerte allí; esto debemos concederlo. Pero debemos ser capaces de centrar el asunto del deber de obedecer las disposiciones jurídicas para la gran mayoría (para los que nacieron allí), si seriamente tenemos la pretensión de que los ciudadanos tienen o pueden tener un deber de conformar su conducta a las disposiciones. En breve, necesitamos ser capaces de discutir el deber que se puede tener de conformarse a las disposiciones del país en el que se ha nacido, sin asumir que el deber existe y que sólo puede existir sobre la base de algún compromiso voluntario o transacción determinada cuya función es poner al ciudadano específicamente bajo ese deber 6 .

Un último punto valioso. A menudo, la gente habla de un deber de obedecer al Derecho para referirse a un sistema específico. Aquí la obligación de obedecer las disposiciones jurídicas no se representa en absoluto como una obligación de tipo moral; sino, más bien, es concebida sobre la base de alguna característica del propio sistema político. Así, se podría alegar que en un Estado democrático las normas de la democracia requieren que se acepte

\footnotetext{
${ }^{6} \mathrm{La}$ aproximación que estoy esbozando en este párrafo ha sido seguida por diferentes teóricos. Véase, por ejemplo, Rawls, Teoría de la justicia (Cambridge, MA: Harvard University Press, ed. Revisada 1999) sec. 19 y cap. 6, esp. Sec. 51-3; R. Martín, A System of Rights (Oxford, Clarendon Press, 1993), cap. 8; también Jeremy Waldron, "Special ties and natural duties", Philosophy and Public Affairs, 22 (1993), y los caps. en Horton y en Dworkin citados en la nota 5. Para una crítica de estas aproximaciones, véase Richard Dagger, "Membership, Fair Play, and Political Obligation", Political Studies, 48 (2000).
} 
el Derecho establecido democráticamente como Derecho, como Derecho obligatorio, y estar dispuesto a cumplirlo mientras permanezca en vigor.

El problema de adoptar la aproximación muy general de las "razones morales" para la obligación política (donde consideramos solamente razones morales a las que obligan a todas las personas en todo tiempo y lugar a obedecer todas las disposiciones jurídicas de su país) no es sólo que no funciona, lo que ya se ha mostrado, sino que además desvía la atención en relación con la noción de obligación especial (del tipo que sea) hacia las disposiciones como tales disposiciones.

Parece que si determinaramos lo que puede decirse simplemente de las disposiciones en tanto que disposiciones y del sistema político específico en el que ellas existen, podríamos en principio generar y justificar tal deber. Si no podemos hacerlo, podríamos preguntarnos si, después de todo, realmente estamos hablando de obligación política. Si queremos tomarnos en serio la cuestión de una obligación hacia las disposiciones en cuanto tales, entonces deberíamos hacer de las razones específicas del sistema nuestra primera línea de ataque en la determinación de los fundamentos de la obligación de obedecer al Derecho.

Permítanme ahora resumir los principales temas de la nueva aproximación sugerida para evaluar la obligación política. Primero, hay que enfatizar el caso de la gente que nace en un país determinado y que reside en él a lo largo de su vida. Y no asumir que cualquier deber de obedecer las disposiciones es un deber que ha sido adquirido voluntariamente o que es implicado por una transacción de algún tipo. Segundo, hay que, al menos en el peldaño inicial, centrarse en las características específicas del sistema político del país en el que esa gente reside, para ver si de estas características surge algún tipo de deber de obedecer allí las disposiciones. Y, finalmente, hay que elevar la cuestión en busca de la generalidad, tratando de encontrar razones generales que respaldasen una obligación de todas las personas en todos los tiempos y lugares de obedecer todas las disposiciones jurídicas de su país.

Seguir esta nueva aproximación recién sugerida no excluye que podamos formularnos las preguntas morales. Podemos seguir cuestionándonos si un sistema de concepciones e instituciones políticas en el que los elementos de la obligación política han sido establecidos como una incrustación puede ser moralmente aceptable. O podemos cuestionar si muchas disposiciones generadas por ese sistema son moralmente aceptables. Esto es lo mismo que preguntar si la obligación política de un sistema específico puede estar moralmente justificada.

Pero las cuestiones que aquí estamos formulando sólo pueden preguntarse y responderse en el orden que les he dado. Sin mostrar primero que la obligación es debida a las disposiciones qua disposiciones y que tal obli- 
gación se le puede dar una justificación sistemática específica, cualquier programa para la justificación moral de la obligación política parecería estar fuera del objetivo. No podría decirnos si (o porque) tenemos un deber de cumplir las disposiciones en tanto que disposiciones. $\mathrm{Y}$ esto olvidaría el punto sobre el que levantar la cuestión de la obligación política en primer lugar. $\mathrm{O}$ así yo lo argumentaría ${ }^{7}$.

\section{Otro intento para la obligación política}

A partir de esta nueva aproximación que he esbozado, podemos brevemente considerar un ejemplo de cómo se desarrollaría. Imaginemos un esquema simplificado de sistema político que incluya unas nociones mínimas tales como derechos constitucionales fundamentales (o derechos civiles básicos) y procedimientos democráticos.

Los derechos civiles activos son derechos políticos universales dentro de una determinada sociedad. Hay maneras de actuar, o maneras de ser tratado, que son específicamente reconocidos y afirmados en el Derecho para todos y cada uno de los ciudadanos de allí (o, en el caso de los límites, para toda persona individual) y son activamente promovidos.

Cuando tales derechos han sido promulgados, aplicados y coordinados entonces tenemos un sistema de reglas de derechos civiles. Y no podríamos tener un tal sistema sin agencias que hicieran esas cosas. A su vez, estas agencias tendrían que mostrar algún grado de coordinación entre ellas. De este modo, el estado, visto como un conjunto coordinado de agencias, entraría en el cuadro como un instrumento para la producción de un sistema de derechos civiles.

Pero ¿cómo podrían las agencias del estado promulgar reglas que constituirían, o al menos ayudarían a definir, los derechos civiles de las personas individuales? ¿Qué bases se podrían tener para creer que las reglas y prácticas generadas por la acción gubernamental fueran realmente reglas de derechos, prácticas de derechos? Esta es una cuestión difícil, pero podría contestarse que es así donde hay democracia.

Plausiblemente podría argumentarse que las instituciones democráticas - elegibilidad universal para votar (una persona, un voto), votación regular y competitiva en dos niveles distintos (el nivel del parlamento y el nivel de elecciones generales) y regla de las mayorías- pueden, actuando como un conjunto (y sobre una base electoral mayoritaria), efectivamente proveer el conjunto requerido por los derechos civiles. Podría pretenderse que los procedimientos democráticos son una forma estable y relativamente confia-

\footnotetext{
${ }^{7}$ Para una elaboración, véase mi libro A System of Rights, cap. 1; y también pág. 186.
} 
ble de identificar, y por tanto de implementar, disposiciones y políticas al servicio del interés común de los votantes o a gran número de ellos, presumiblemente al menos de la mayoría.

Los derechos civiles identifican formas de actuar o formas de ser tratado que podrían exigirse por todas las personas para ellas mismas individualmente. Exigirse porque estas formas de actuar (o de ser tratados) forman parte de lo que beneficia a cada persona o son instrumentales para ello.

La gente ve estas maneras como medios para o parte de cosas que considera valiosas. Todos preferirían tener a su disposición estas formas que no tenerlas -incluso bajo la condición de que esta misma forma esté a disposición de otros, en realidad, de todos. Una manera de actuar (o de ser tratado) que ha sido identificada y sostenida por una disposición y que puede ser apoyada, argumentadamente, como en el interés de todos, en el interes de todos y cada uno de los ciudadanos, está justificada como un derecho civil.

A menudo se dice que los derechos, ciertamente los derechos justificados, son correlativos de deberes, significando de este modo que un derecho siempre implica o tiene adherido algún deber de otros. El punto aquí, puesto precisamente, es que cualquier derecho genuino debe implicar algún significante normativo de guía de la conducta de personas distintas del titular. Y es esta verdad, crucial para el concepto de derechos, la que quiero enfatizar.

Así, por cada derecho (por cada manera de actuar o de ser tratado establecida sobre la parte un titular de un derecho) hay algún tipo de significante de guía normativa dada para la conducta de otras personas. En un sistema de derechos civiles, los derechos de los individuos se mantienen, al menos en parte, por la acción de la gente sobre la que recae el deber relevante (la guía normativa relevante), sobre estas personas recaen las directivas primarias de conducta que han sido impuestas. A veces un determinado requerimiento se impone a todos los ciudadanos (ej. la prohibición del asesinato). Otras veces el "deber" requerido se impone sólo sobre algunos.

Más allá de toda la gama de casos, lo importante es que cada ciudadano tiene hacia los otros ciudadanos (todos son titulares de derechos) el estatus de titular de un deber en el caso de muchos derechos civiles. Y cada titular de un derecho puede reclamar del estado, o a los otros, ayuda para mantener estos derechos - para hacer cumplir estos derechos- cuando los titulares primarios del deber dejan de actuar correctamente, actúan desviadamente. Así, conforme al argumento, todo ciudadano individual tiene algunos -aunque diferentes- requerimientos conducta impuestos por los derechos civiles.

En último término, si asumimos que las principales instituciones democráticas están dadas (sufragio universal, competencia electoral y regla de las mayorías) y que las disposiciones de derechos civiles han sido debidamente establecidas bajo estas instituciones; consecuentemente, es aplicable la pre- 
sunción de que estas disposiciones incorporan un beneficio de todos y cada uno de los ciudadanos en las diferentes maneras de actuar, o de ser tratados, que el Derecho garantiza para todas las personas en general.

No estoy sugiriendo, por cierto, que las instituciones democráticas siempre alcancen el resultado identificado. Ciertamente no es así en cada caso. Pero lo importante es que si las instituciones democráticas se ajustan a aquello que las justifica en primer término, entonces en efecto tienden a producir normas de derechos civiles (entre otras cosas) y no a actuar reemplazando o perjudicando tales derechos ${ }^{8}$.

Donde los derechos están establecidos en disposiciones democráticas (como lo están en el sistema de derechos civiles que estamos investigando), es precisamente en las disposiciones que establecen las directivas relevantes para la conducta de las personas en respeto de tales derechos. Obviamente, los derechos no cuentan para mucho a menos que sean mantenidos. Y la principal manera de mantenerlos es que la gente cumpla sus deberes y actue en conformidad con las disposiciones de derechos de derechos civiles. Se sigue, entonces, que el miembro típico (o ciudadano) en tal sociedad tiene deberes definidos de conformar su conducta a las disposiciones que definen derechos y mantienen derechos. Allí donde los derechos están definidos y mantenidos por el Derecho, cumplir con el deber de uno bajo un determinado derecho consiste simplemente en cumplir la(s) disposición(es) en cuestión.

Así, el ciudadano típico en un sistema democrático de derechos tiene allí un deber peculiar o especial, el deber de obedecer las disposiciones de derechos civiles. Este no es un deber hacia las disposiciones qua disposiciones; podemos suponer que en esta sociedad hay disposiciones válidas que no caerían bajo el deber en cuestión. Más bien, el deber de adaptarse al Derecho es debido precisamente respecto de las disposiciones promulgadas que definen o mantienen derechos civiles. En el caso más simple, este deber es un deber sistemático específico donde mantiene el bien para cada ciudadano.

Las disposiciones sobre derechos son obligatorias sobre la conducta no porque las disposiciones impliquen derechos, sino porque son derechos. Por tanto, no es que cada una sea conforme a las normas de derechos aplicables por lo que están afirmadas en disposiciones, hacia las que, independientemente de este carácter de derechos, los ciudadanos tienen una posición de

\footnotetext{
${ }^{8}$ La conexión de estos dos principales elementos -derechos civiles e instituciones políticas democráticas- está desarrollada y argumentada, de manera considerablemente extensa, en mi libro System of Rights, especialmente en los caps. 6,7,12 y el apéndice. Para un conveniente tratamiento del principal argumento, véase mi artículo más breve "Basic Rights", Rechtstheorie, Beiheft vol. 15 (1993).
} 
deber; es, más bien, la inversa. Los ciudadanos son requeridos a obedecer las disposiciones de derechos -y ellos mismos comprender correctamente estar bajo un tal requerimiento- en tanto que aquellas disposiciones establecen correctamente (como podemos presumir que hacen) las guías normativas relevantes para la conducta apropiada hacia los derechos implicados.

Los ciudadanos tendrían una posición comprometida a atenerse a aquellas disposiciones. Resultaría claro, por otra parte, que los derechos de cada persona estarían apoyados por la conducta obediente a las disposiciones que son derechos que aquella persona tiene. Así, la reciprocidad de un sistema de derechos civiles, y la idea de fondo de que los derechos civiles justificados son beneficiosos para todos y cada uno, provee la principal razón o justificación de los ciudadanos para cumplir su deber mediante la obediencia a las disposiciones de derechos.

Si la idea de un sistema de derechos requiere cumplimiento de todas sus disposiciones o si sólo implica estricto cumplimiento de todas las disposiciones de derechos civiles es claramente una cuestión disputada. Pero tengo mis dudas de que haya alguna disputa real dado que la posición de los ciudadanos hacia el Derecho en este particular sistema es especial, una posición que los adhiere a las disposiciones, esto es, a las disposiciones que formulan y ayudan a mantener derechos civiles.

Nuestro análisis satisface los estándares que pusimos antes para cualquier nueva y mejorada teoría de la obligación política. En el desarrollo de este análisis no hemos intentado encontrar razones generales que asegurasen una obligación de toda la gente en todos los tiempos y lugares de obedecer las disposiciones jurídicas de su país. Mas bien, nos hemos centrado a través de una razón sistemática específica, dada por la idea de un sistema democrático de derechos civiles, diciendo que los ciudadanos tienen allí un deber de obedecer las disposiciones de derechos. Este particular deber no es uno que nosotros asumimos por haber sido voluntariamente adoptado, ni se ha extraido de alguna forma de transacción. Y, finalmente, hemos enfatizado y tomado en cuenta los casos de la gente que simplemente ha nacido en un determinado país o que han sido residentes durante toda su vida; ellos son los progenitores primarios del tal sistema de derechos civiles en aquel país, así como ellos son sus beneficiarios primarios.

Lo que hemos estado buscando, a través de este análisis, son razones específicas para que un determinado sistema político pudiera obligar a la gente a conformar su conducta a las disposiciones jurídicas, simplemente como tales disposiciones de ese sistema. Si un sistema así existe realmente en un grado apreciable en el país en el cual vive un grupo de personas, entonces allí habremos encontrado razones que obligarán a esa gente respecto de las disposiciones, simplemente en tanto que disposiciones correctamente 
Rex Martin

dictadas, o que obligarán a la gente con respecto a un importante subconjunto de esas disposiciones. Siguiendo las líneas de esta nueva aproximación alcanzaremos una noción de obligación política definida y útil.

(Trad. de Josep Aguiló Regla) 